\title{
Monitoring Nitric Oxide-Induced Hypoxic Tumor Radiosensitization by Radiation-Activated Nanoagents under BOLD/DWI Imaging
}

Yan Dou, ${ }^{\dagger, \#, *}$ Fangshi Zhao, ${ }^{\dagger, \#}$ Xue Li, ${ }^{\ddagger}$ Yanyan Guo

$\uparrow$ Department of Radiology and Tianjin Key Laboratory of Functional Imaging,

Tianjin Medical University General Hospital, Tianjin 300052, P.R. China

* Department of Radiology and Department of Radiation Oncology, Tianjin Medical University Second Hospital, Tianjin 300211, P.R. China

\# These authors contributed equally to this work.

*E-mail: douyan@tmu.edu.cn (Y. Dou).

the number of pages: 10

the number of figures: 9 


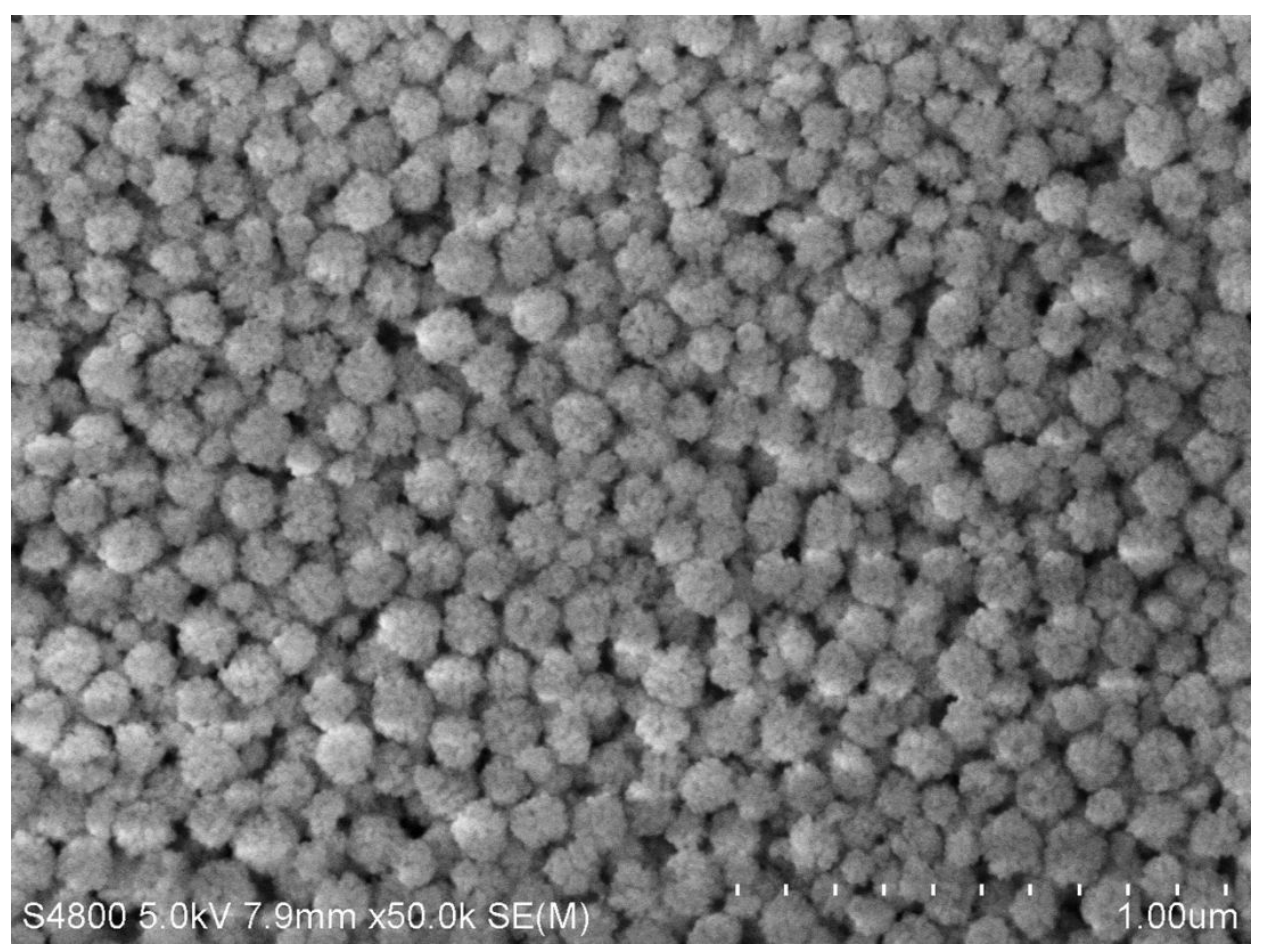

Figure S1. SEM images of $\mathrm{NSC} @ \mathrm{SiO}_{2}-\mathrm{SNO}$ nanoagent. 


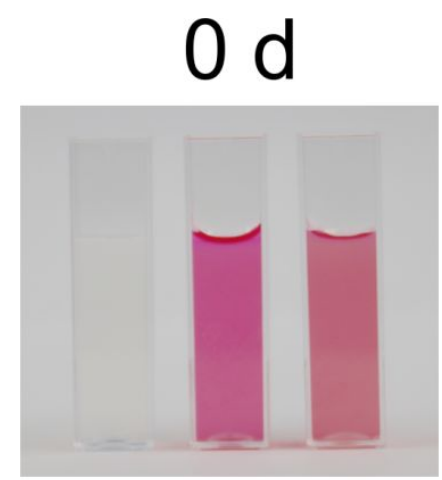

(1) (2) (3)

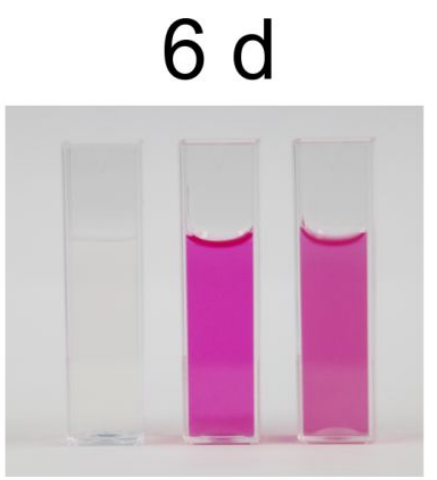

(1) (2) (3)

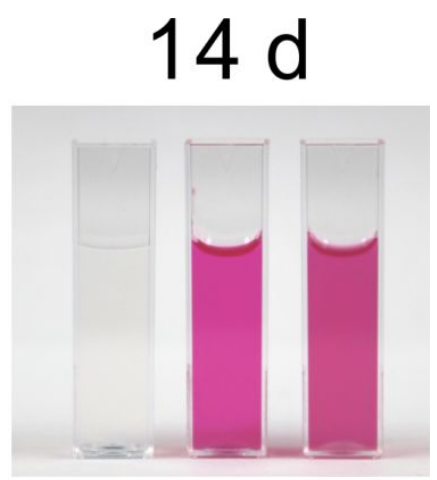

(1) (2) (3)

Figure S2. The photographs taken at different time points $(0,6 \mathrm{~d}$, and $14 \mathrm{~d})$ during this nanoagent incubated in different biological media. (1) PBS; (2) DMEM; (3) DMEM+10\% FBS. 


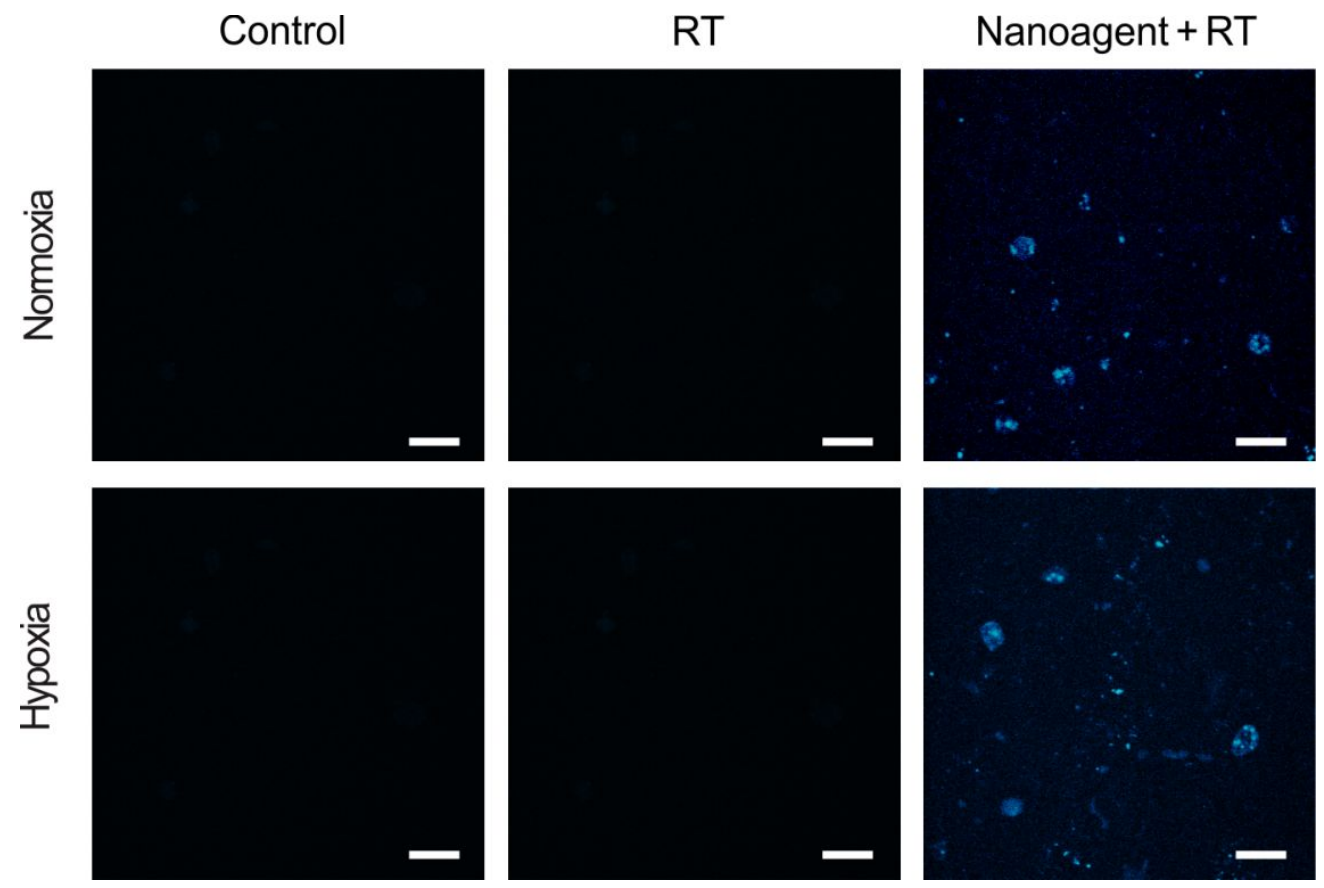

Figure S3. Immunofluorescence staining of $4 \mathrm{~T} 1$ cells after different treatments by DAF-FM DA staining $($ Scale bars $=50 \mu \mathrm{m})$. 

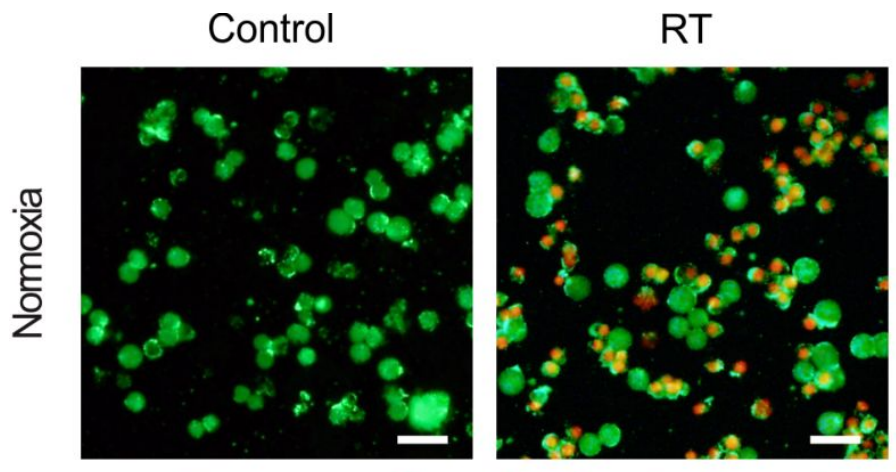

$$
\text { Nanoagent + RT }
$$
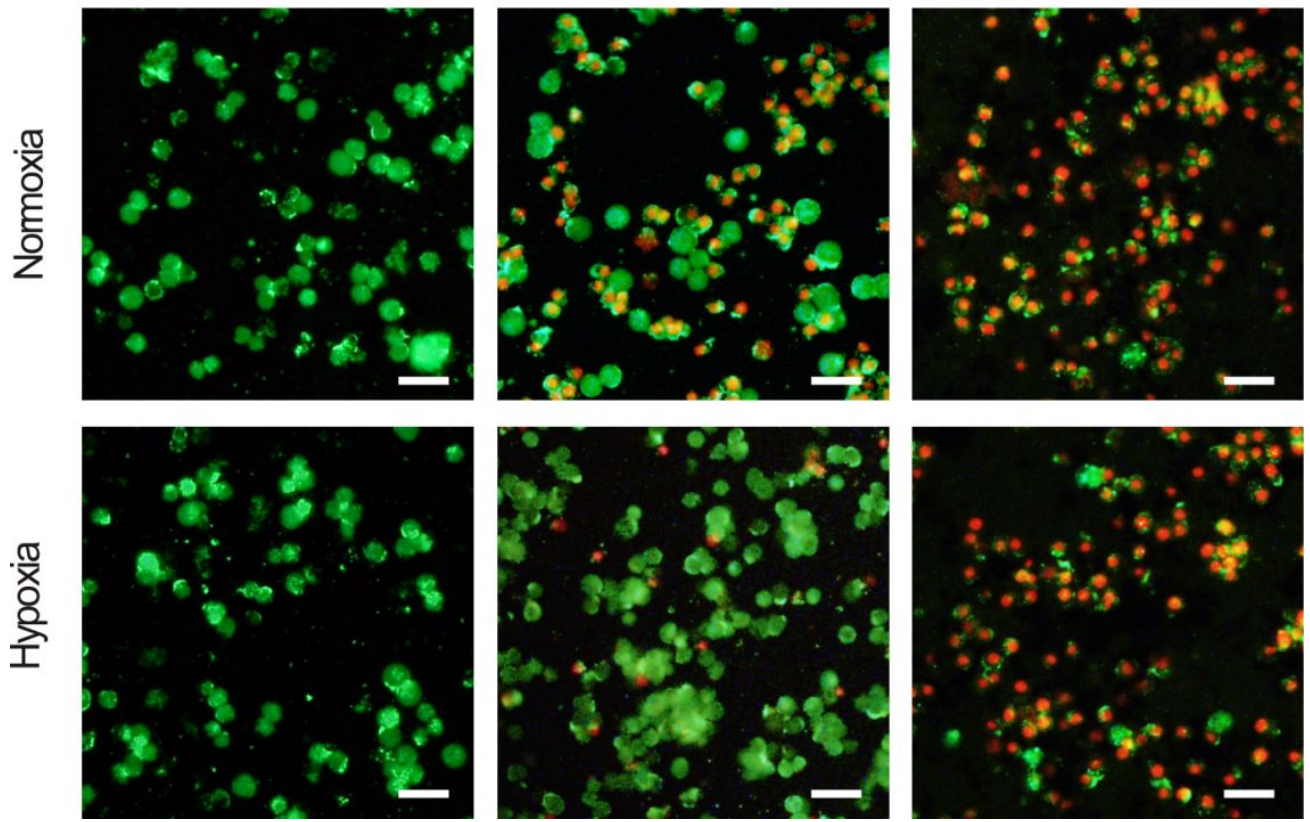

Figure S4. Immunofluorescence staining of 4T1 cells after different treatments by

Calcein-AM/PI staining (Scale bars $=50 \mu \mathrm{m})$. Green: Live. Red: Dead. 


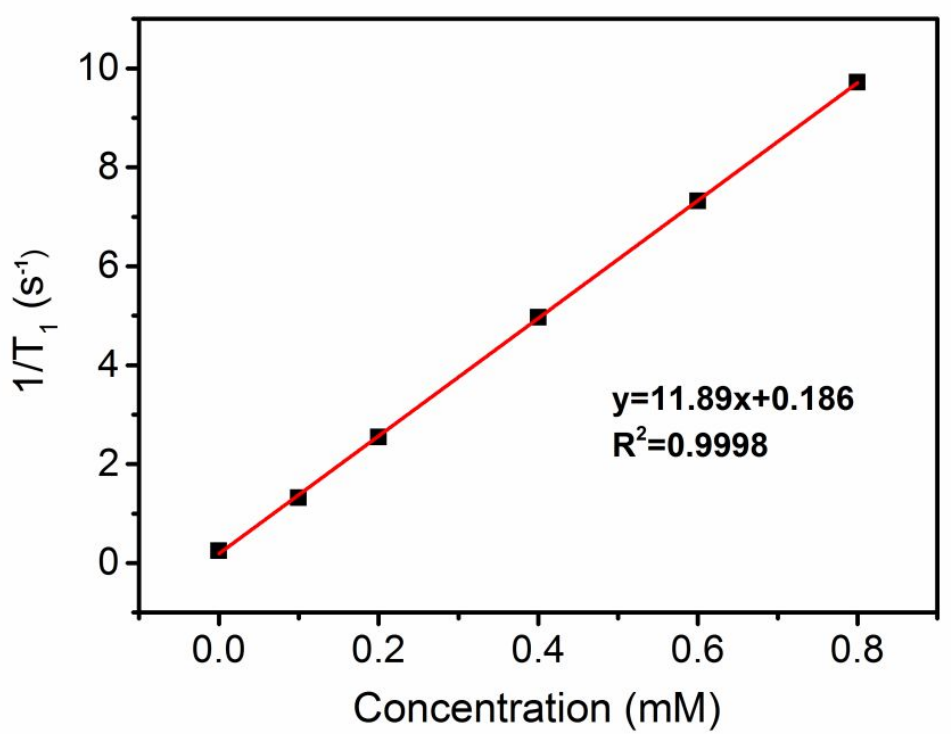

Figure S5. Longitudinal relaxation rates $\left(1 / \mathrm{T}_{1}\right)$ of the nanoagent vs. Gd concentration. 
a
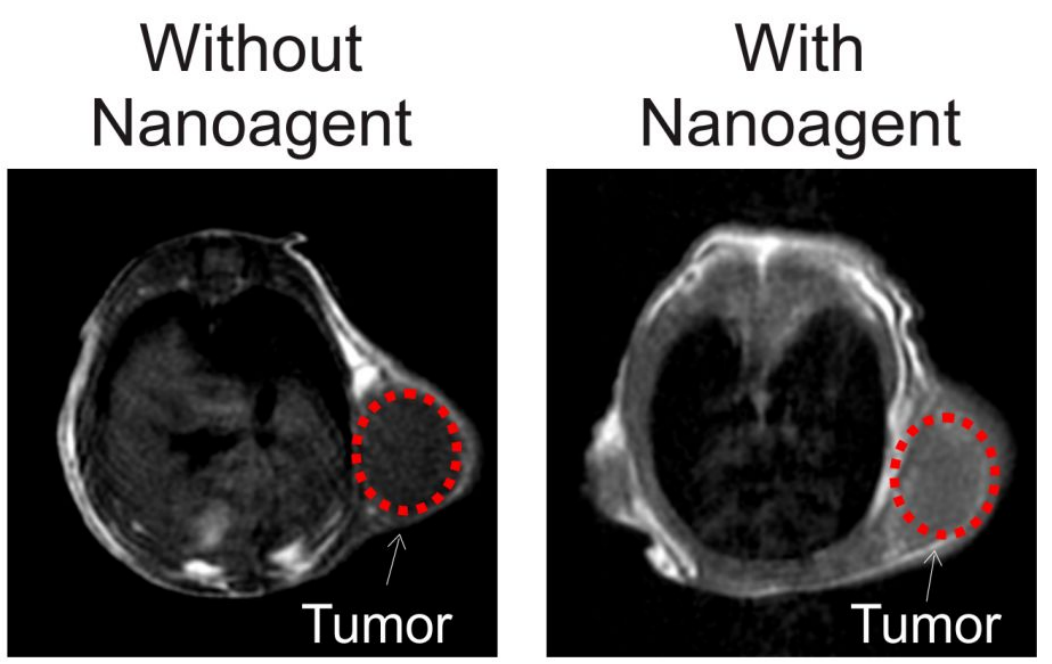

b

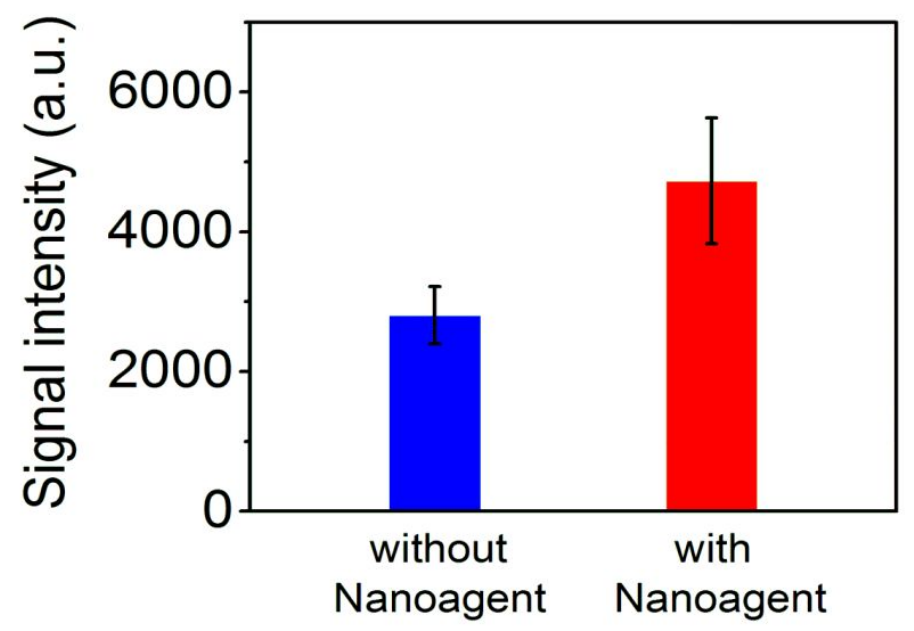

Figure S6. (a) In vivo $\mathrm{T}_{1}$-weighted $\mathrm{MR}$ imaging and (b) quantitative analysis of signals in tumor of mice after $24 \mathrm{~h}$ intravenous injection of the nanoagent. 


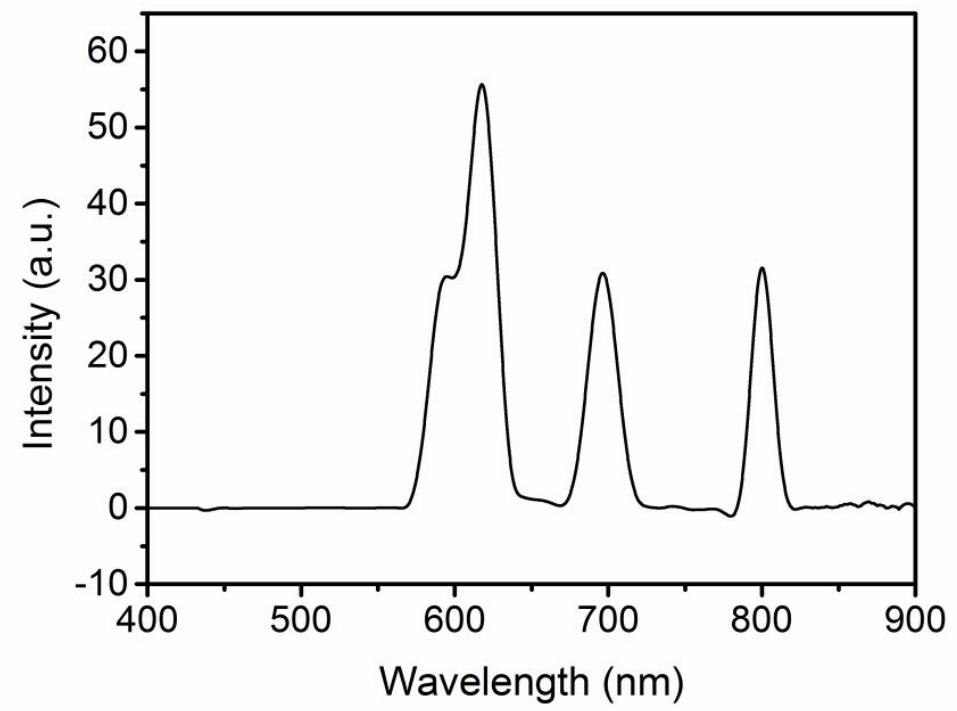

Figure S7. UV-excited photoluminescence spectra of the nanoagent. 


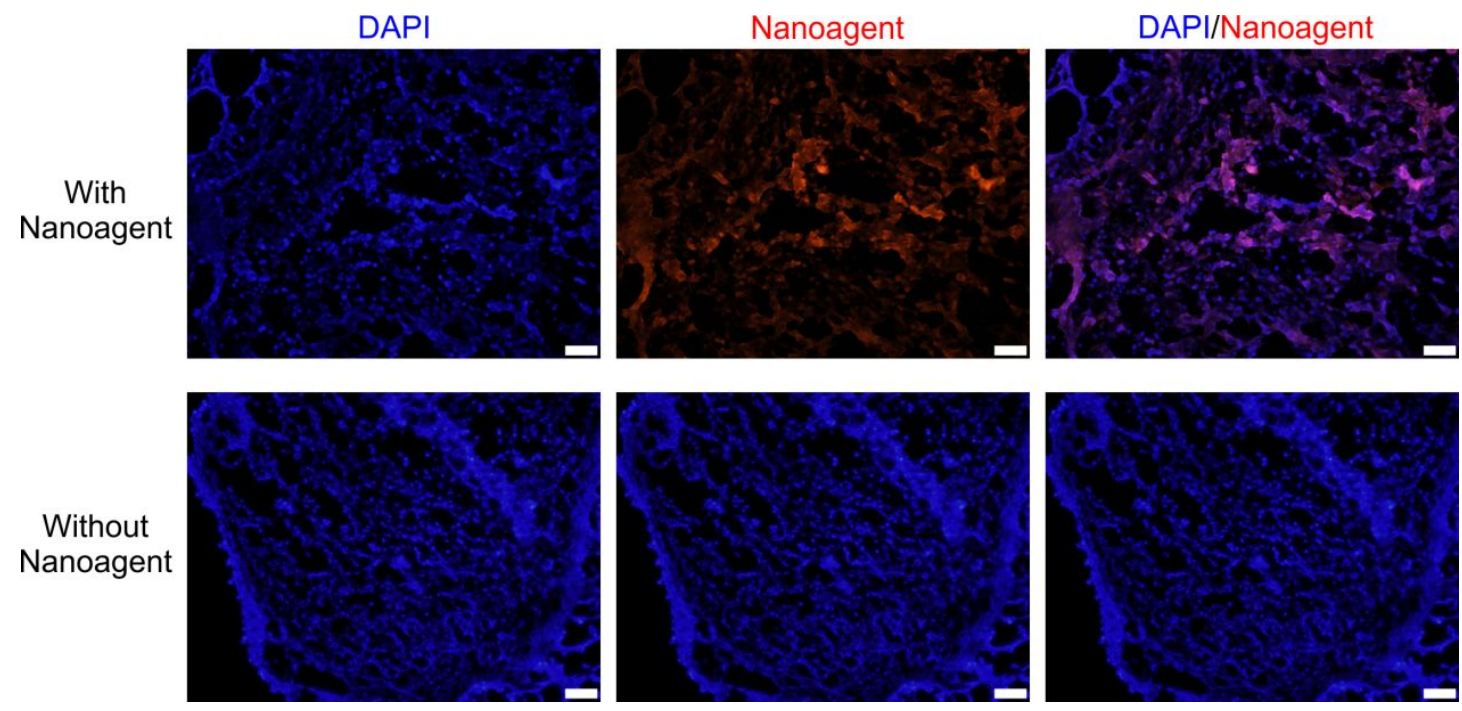

Figure S8. Ex vivo immunofluorescence images of tumor slices from the mice after $24 \mathrm{~h}$ intravenous injection of this nanoagent (Scale bars $=50 \mu \mathrm{m})$. 


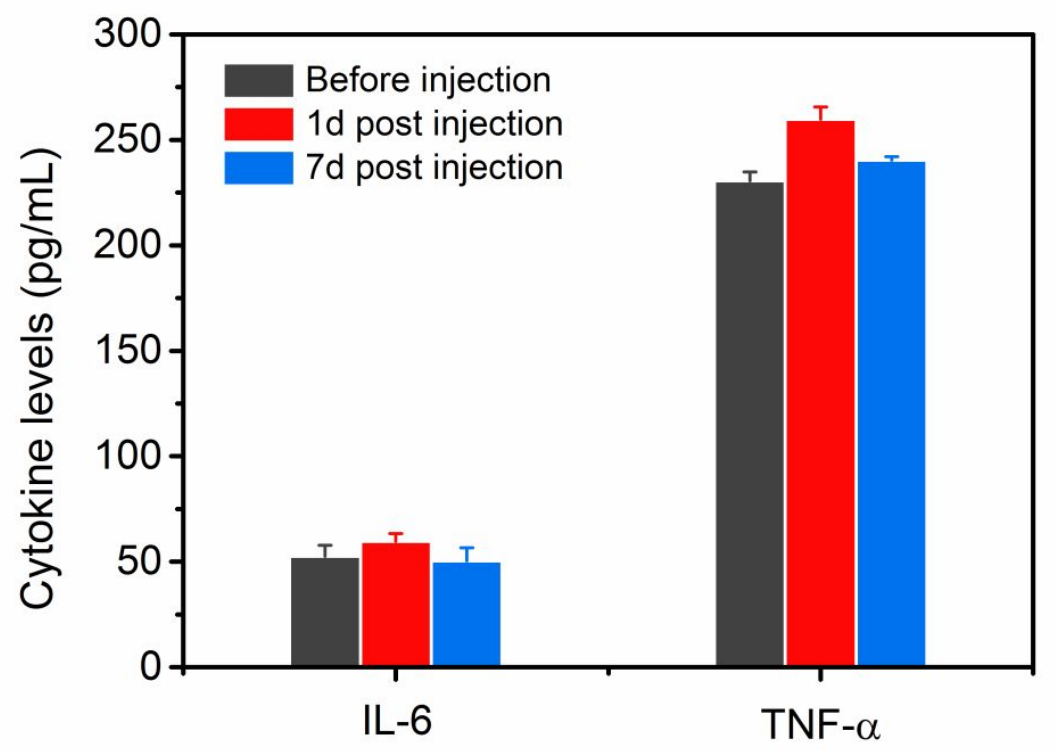

Figure S9. The levels of IL-6 and TNF- $\alpha$ in the blood of mice after injection of the nanoagent by ELISA. 